\title{
The Basoph8 Mice Enable an Unbiased Detection and a Conditional Depletion of Basophils
}

\author{
Christophe Pellefigues ${ }^{*}$, Palak Mehta, Melanie Sarah Prout, Karmella Naidoo, \\ Bibek Yumnam, Jodie Chandler, Sally Chappell, Kara Filbey, Mali Camberis and \\ Graham Le Gros
}

The Malaghan Institute of Medical Research, Victoria University, Wellington, New Zealand

\section{OPEN ACCESS}

Edited by: Jagadeesh Bayry,

Institut National de la Santé et de la

Recherche Médicale (INSERM), France

Reviewed by:

Matthias Mack

University Medical Center

Regensburg, Germany

Elia Tait Wojno,

Cornell University, United States

*Correspondence:

Christophe Pellefigues christophe.pellefigues@ protonmail.com

Specialty section: This article was submitted to

Molecular Innate Immunity,

a section of the journal

Frontiers in Immunology

Received: 26 May 2019 Accepted: 27 August 2019 Published: 10 September 2019

Citation:

Pellefigues C, Mehta P, Prout MS, Naidoo K, Yumnam B, Chandler J, Chappell S, Filbey K, Camberis M and Le Gros G (2019) The Basoph8 Mice Enable an Unbiased Detection and a Conditional Depletion of Basophils.

Front. Immunol. 10:2143.

doi: 10.3389/fimmu.2019.02143
Basophils are granulocytes involved in parasite immunity and allergic diseases, known for their potent secretion of type 2 cytokines. Identifying their functions has proven to be controversial due to their relative rarity and their complex lineage phenotype. Here, we show that the expression of basophils lineage markers CD200R3 and $F_{C \varepsilon R l} \alpha$ is highly variable in inflammatory settings and hinders basophils identification by flow cytometry across multiple disease states or tissues. Fluorophore-conjugated antibody staining of these lineage markers strongly activates basophil type 2 cytokine expression, and represents a potential bias for coculture or in vivo transfer experiments. The Basoph8 is a mouse model where basophils specifically express a strong fluorescent reporter and the Cre recombinase. Basophils can be identified and FACS sorted unambiguously by their expression of the enhanced yellow fluorescent protein (eYFP) in these mice. We show that the expression of the eYFP is robust in vivo during inflammation, and in vitro on living basophils for at least $72 \mathrm{~h}$, including during the induction of anaphylactoid degranulation. We bred and characterized the Basoph8xiDTR mice, in which basophils specifically express eYFP and the simian diphtheria toxin receptor (DTR). This model enables basophils conditional depletion relatively specifically ex vivo and in vivo during allergic inflammation and their detection as eYFP+ cells. In conclusion, we report underappreciated benefits of the commercially available Basoph8 mice to study basophils function.

\section{Keywords: basophil, Basoph8, depletion, flow cytometry, phenotype}

\section{INTRODUCTION}

Basophils are potent blood granulocytes known to be associated with Type 2 immune responses. They are notably involved in helminths and ticks protective immunity, and in the development of some allergic and autoimmune responses (1). As for murine mast cells, basophils are characterized by their expression of the high affinity receptor for $\operatorname{IgE}(\mathrm{FcERI} \alpha)$, and of CD200R3, an activating receptor of the CD200R receptor-like family (2). Efforts to define their function using depleting monoclonal antibodies targeting basophil "lineage defining" surface antigens such as FceRI $\alpha$ and CD200R3 have proven to be controversial. This is likely due to the lack of specificity of these antibodies and to the fact that they can lead to a functional activation of basophils in vivo, as much as to their depletion (2-9). The MARI antibody targeting FceRI $\alpha$ was shown to activate basophils IL-4 and histamine secretion both ex vivo and in vivo (8). Similarly, Ba13 targeting CD200R3 was 
shown to induce basophils IL-4 secretion in vitro and anaphylactoid symptoms in vivo (2). These antibodies are commonly used to FACS sort basophils for in vivo transfer experiments $(10,11)$, however the activation of basophils induced by the sorting process has never been addressed.

Strategies to specifically and conditionally deplete basophils have been developed using the promoter regions of genes specifically expressed by basophils, such as MCPT8 (Mast Cell Protease 8). The MCPT8-DTR (Diphtheria Toxin Receptor) mouse show a very potent depletion of basophils lasting 6 days after one intraperitoneal injection of Diphtheria Toxin (DT) (12). However, recently, El Hachem et al. observed that the injection of high doses of DT in these mice also resulted in a depletion of neutrophils and eosinophils (13) which was linked to the transient expression of MCPT8 in granulocyte macrophage progenitors (GMPs). An other strategy to conditionally deplete basophils has been developed as the Bas-TRECK mice by targeting enhancers of the il4 gene shown to be specifically functional in basophils at steady state (14). However, IL-4 can be expressed by numerous cell types during allergic inflammation, including some subsets of CD4 ${ }^{+} \mathrm{T}$ cells, mast cells, NKT cells, $\gamma \delta \mathrm{T}$ cells, neutrophils, eosinophils, macrophages, and ILC2s (15-20). It remains to be seen if some of these subsets would also be depleted in the Bas-TRECK mice in conditions where the il4 locus would be in an open state, as the regulation of the expression of IL-4 is complex and still ill-defined in some cell types.

The Basoph 8 mouse model has been generated by inserting a sequence coding for the enhanced Yellow Fluorescent Protein (eYFP)-IRES-Cre recombinase, immediately after the $5^{\prime}$ promoter and untranslated (UTR) region of the MCPT8 gene, effectively knocking out its expression (21). The expression of eYFP by basophils proved to be sufficient for their tracking in vivo by two photon microscopy and flow cytometry (22). Later studies confirmed that the expression of the eYFP was restricted to basophils among mature hemopoietic cell types (23). Very recently, Shibata et al. described a new MCPT8-iCre mouse using a similar design strategy as the Basoph8, inserting the Cre recombinase coding sequence as a knock-in in the first exon of MCPT8 (24). Crossing these mice with the Rosa-eYFP mice showed that Cre mediated recombination affected $\sim 15 \%$ of eosinophils and $\sim 7 \%$ of neutrophils $(23,24)$. These results showed that the non-specific activity of the MCPT8 driven Cre recombinase seems rather limited in steady state conditions.

Here, we show that basophils expression of the lineage markers FceRI $\alpha$ and CD200R3 is deeply downregulated during helminth infection and skin allergic inflammation, respectively. Basophils also showed a time dependent expression of Ly6C in vivo during allergic inflammation, which could be recapitulated by a stimulation ex vivo with IL-3. Basophils lineage markers FceRI $\alpha$ and CD200R3 are both known to be potent activators of basophils upon crosslinking in vivo. In order to find the best strategy to FACs sort basophils without activating them, we analyzed the activation status of Basoph8x4C13R (B8x4C13R) basophils stimulated with either MARI (anti-FceRI $\alpha$ ) or Ba13 (anti-CD200R3), and found out that basophils were potently activated when incubated with any of these antibodies. To bypass these biases, we used the Basoph8 mice, in which basophils could specifically be FACS sorted based on their strong expression of the eYFP, without any artificial antibody mediated activation. Basoph8's eYFP expression was strong and allowed an unambiguous identification of basophils in all inflammatory conditions and tissues tested in vivo. It was also robust, as it was unaltered by anaphylactoid degranulation, or for at least $72 \mathrm{~h}$ on FACS sorted living basophils ex vivo. We then bred the Basoph8x iDTR (B8xiDTR) mouse to enable the conditional depletion of basophils in mice expressing a strong specific reporter. The iDTR mice express the simian DTR only in cells showing Cre recombinase activity (25). We evaluated the specificity of the DTR expression and of basophils depletion both ex vivo and in vivo to show that this model was relatively specific to basophils during steady state. The B8xiDTR mice also enabled a short-term depletion of basophils in different models of allergic inflammation. We conclude that the Basoph8 mouse is an underappreciated tool that can be used for both an ideal identification and for a conditional depletion of basophils.

\section{RESULTS}

\section{The Basophils "Lineage" Surface Phenotype Is Altered in Inflammatory Conditions}

Basophils from naïve mice are usually described as $\mathrm{CD}_{4} \mathrm{~b}^{+}$

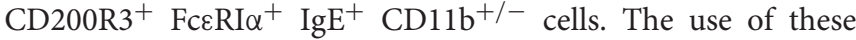
lineage markers can be problematic as they are also expressed by mast cells and certain subsets of inflammatory monocytes or dendritic cells $(4,7)$. In order to define the most robust phenotype of basophils, we analyzed the surface expression of these markers on Basoph8 (B8) eYFP+ cells across various inflammatory conditions.

Heligmosomoides polygyrus ( $\mathrm{Hp})$ is a murine intestinal helminth that induces a potent peripheral basophilia, peaking at day 15 post infection (26). As previously reported, the detection of the expression of FceRI $\alpha$ on the surface of basophils is impaired during $\mathrm{Hp}$ infection (27). However, surface bound IgE was barely detected on basophils from naïve mice, but increased several-fold after helminth infection (Figure 1A). This analysis shows that the variability of the detection of FceRI $\alpha$ and IgE hinders basophils identification during the course of a helminth infection.

We also analyzed the phenotype of skin infiltrating basophils in a model of MC903 induced atopic dermatitis (28). The identification of leucocytes in non-lymphoid tissues commonly relies on the detection of CD45 expression. However, basophils express low levels of CD45 in steady-state conditions, which can impair their detection as $\mathrm{CD} 45^{+}$leucocytes in non-lymphoid tissues such as the skin. Additionally, a low CD45 expression is commonly used to distinguish basophils from others leucocytes (29). In accordance with this, we observed extremely low levels of CD45 expression on the surface of $\mathrm{eYFP}^{+}$basophils from the skin of naïve mice, but this expression was potently increased upon MC903-induced dermatitis (Figure 1B). The expression of CD200R3 is shared between basophils and mast cells, and 


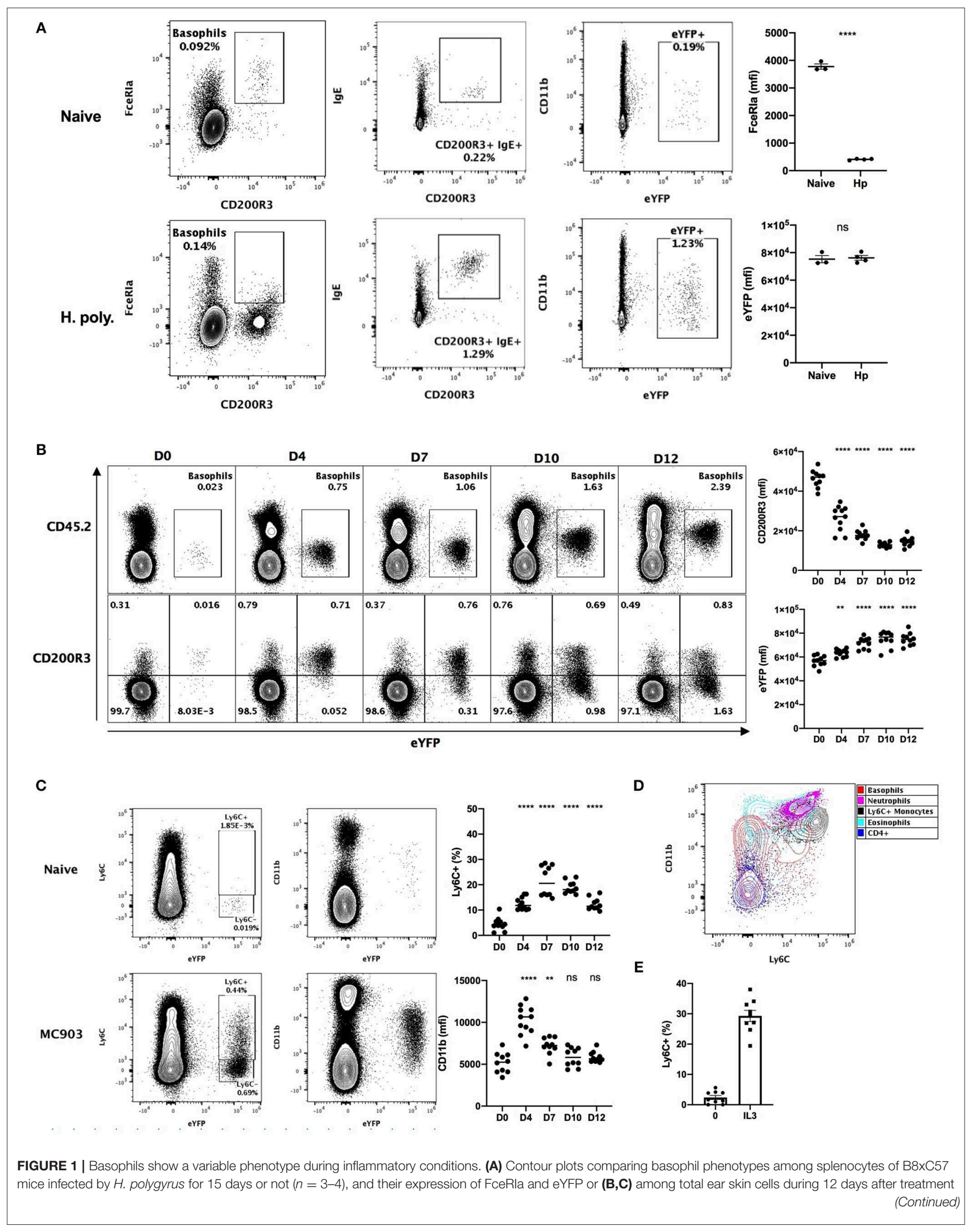


FIGURE 1 | with MC903 $(n=10-11)$. (B) The expression of CD200R3 and eYFP by eYFP+ basophils is depicted overtime. (C) The expression of CD11b by and the proportion of Ly6C+ eYFP+ basophils is shown (D) Overlay of eYFP+ basophils and CD45+ leucocytes including, CD3+ CD4+ T cells (CD4+), CD11b+ Ly6G+ Neutrophils, SSChi SiglecF+ Eosinophils, CD64lo Ly6G- SiglecF- eYFP- CD11b+ Ly6C+ inflammatory monocytes (Ly6C+ monocytes). (E) Proportion of eYFP+ basophils expressing Ly6C after $24 \mathrm{~h}$ of stimulation of whole splenocytes from Basoph8 mice with $1 \mathrm{ng} / \mathrm{mL}$ of IL3 $(n=9)$. Data are representative of 2 (B-E) or 3 (A) independent experiments giving similar results. Statistics are unpaired $t$-test (A) or one-way ANOVA with a Dunnett's comparison to DO (B,C). Ns, Non-significant; ${ }^{* *} p<0.01 ;{ }^{* * *} p<0.0001$.

is considered to be a robust marker for basophils detection (2). However, we found that basophils CD200R3 expression was strongly downregulated on $\mathrm{eYFP}^{+}$basophils in the skin in atopic conditions (Figure 1B). This confirms previous reports showing a strong decrease of basophils CD200R3 expression in some allergic models (30). These results suggest that neither $\mathrm{CD} 45^{\text {low }}$ or CD200R3 expression are robust or reliable markers for identifying basophils in the skin during allergic inflammation.

We unexpectedly observed a time dependent expression of Ly6C by up to $30 \%$ of $\mathrm{eYFP}^{+}$basophils infiltrating the skin in these atopic conditions, but not in steady state. Similarly, basophils expression of the myeloid marker CD11b was variable along the course of this disease (Figure 1C). Ly6C and CD11b are lineage markers used to identify inflammatory monocytes, eosinophils, and neutrophils. We found that the phenotype of eYFP+ Ly6C + basophils was overlapping with those of Ly6C + monocytes in the atopic skin (Figure 1D). A proper distinction between basophils and monocytes is important as monocyte-derived inflammatory dendritic cells expressing both Ly6C and basophils markers have been described during allergic inflammation (4). Basophils are known to be potently and selectively activated by IL-3 (27), so to confirm the specificity of the expression of Ly6C by mouse basophils we stimulated whole splenocytes from the Basoph8 mice with IL-3. Indeed, the expression of Ly6C on $\mathrm{eYFP}^{+}$basophils could be induced dose-dependently by IL-3 ex vivo (Figure 1D, Supplementary Figure 1), which confirm that Ly6C expression is a feature of a subset of activated murine basophils.

So, basophils show important changes in the expression of their common lineage markers Fc\&RI $\alpha$ and CD200R3 during helminth infection and atopic inflammation, respectively. They also show a very dynamic phenotype that could hinder their identification and their discrimination from other myeloid cells such as inflammatory monocytes. Importantly, the expression of eYFP by basophils seems robust in vivo as it was not downregulated in these inflammatory conditions, but rather upregulated during skin allergic inflammation (Figure 1B), and it always allowed us to unambiguously identify basophils even in an autofluorescent tissue such as the skin (Figures 1A-C).

\section{Basoph8's eYFP Expression Is Robust}

Basophils from the Basoph8 mouse can be identified specifically by their strong eYFP expression in vivo $(21,23)$. However, basophils show dramatic changes of their phenotype during allergic inflammation, and especially during piecemeal or anaphylactic degranulation (31), and the stability of the expression of the eYFP during basophils degranulation has never been addressed. We stimulated whole splenocytes from the Basoph8 mice with concentrations of secretagogues able to induce degranulation for $2 \mathrm{~h}$, as evidenced by a strong increase of the surface expression of the tetraspannin CD63 by most eYFP+ basophils (Figure 2A) (32). This was not associated with any reproducible significant change in basophils eYFP expression, indicating that eYFP expression seems to withstand their degranulation (Figure 2B).

In the Basoph8 mice, eYFP expression arise on bone marrow basophils, and can then be detected on circulating mature basophils (21). However, it is not known if the expression of the eYFP by mature basophils would fade overtime. To answer this question, we FACS sorted basophils as eYFP+ cells from the spleens of Hp infected Basoph8 mice, and stimulated them ex vivo with IL-3 or ionomycin. As expected, living basophil numbers quickly decreased without a source of prosurvival factors such as IL-3 (Figure 2C). The expression of eYFP on unstimulated living basophils (identified as DAPIeYFP+ cells) did not decrease significantly during the first $72 \mathrm{~h}$, but it did significantly increase at 24 and $48 \mathrm{~h}$ after IL-3 and ionomycin stimulation (Figure 2D, two-way ordinary ANOVA with Dunett's post-test, $24 \mathrm{~h} p<0.001,48 \mathrm{~h} p<0.05)$. It is worth mentioning that the number of DAPI+ cells increased during that time, but that we never observed eYFP+ DAPI+ double positive events (Figure 2E). This indicates that basophils are losing the eYFP expression during the apoptotic process.

Identifying basophils as DAPI- CD49b+ CD200R3 + cells in these experiments revealed a minor significant decrease of eYFP expression by basophils left unstimulated for $72 \mathrm{~h}$ (Figure 2F, two-way ordinary ANOVA with Dunett's post-test, $p<0.0001$ ). This was not due to a decrease of the expression of eYFP by most surviving basophils, but rather by an accumulation of rare eYFPand eYFPlo events (Figure 2G). These differences seemed due to the high numbers of apoptotic or dead basophils accumulating in these conditions.

We conclude that the expression of eYFP by basophils is robust, as it is not significantly downregulated during allergic inflammation or infection in vivo (Figure 1), nor by induced anaphylactic degranulation, or during the first $72 \mathrm{~h}$ of basophils lifespan (Figures 2B,D). We underline that the activation of basophils by IL-3 and ionomycin was accompanied by an upregulation of their eYFP expression, which could indicate an increase of the expression of the MCPT8 gene.

\section{Basophils Are Strongly Activated by Antibody Mediated FACS Staining}

The conventional identification of basophils by flow cytometry involves a first step of blocking Fc receptors by using the 2.4G2 rat IgG ("FcBlock"). Single cell suspensions are then stained with a combination of fluorophores conjugated antibodies directed against lineage markers including FceRI $\alpha$ (MAR1) 


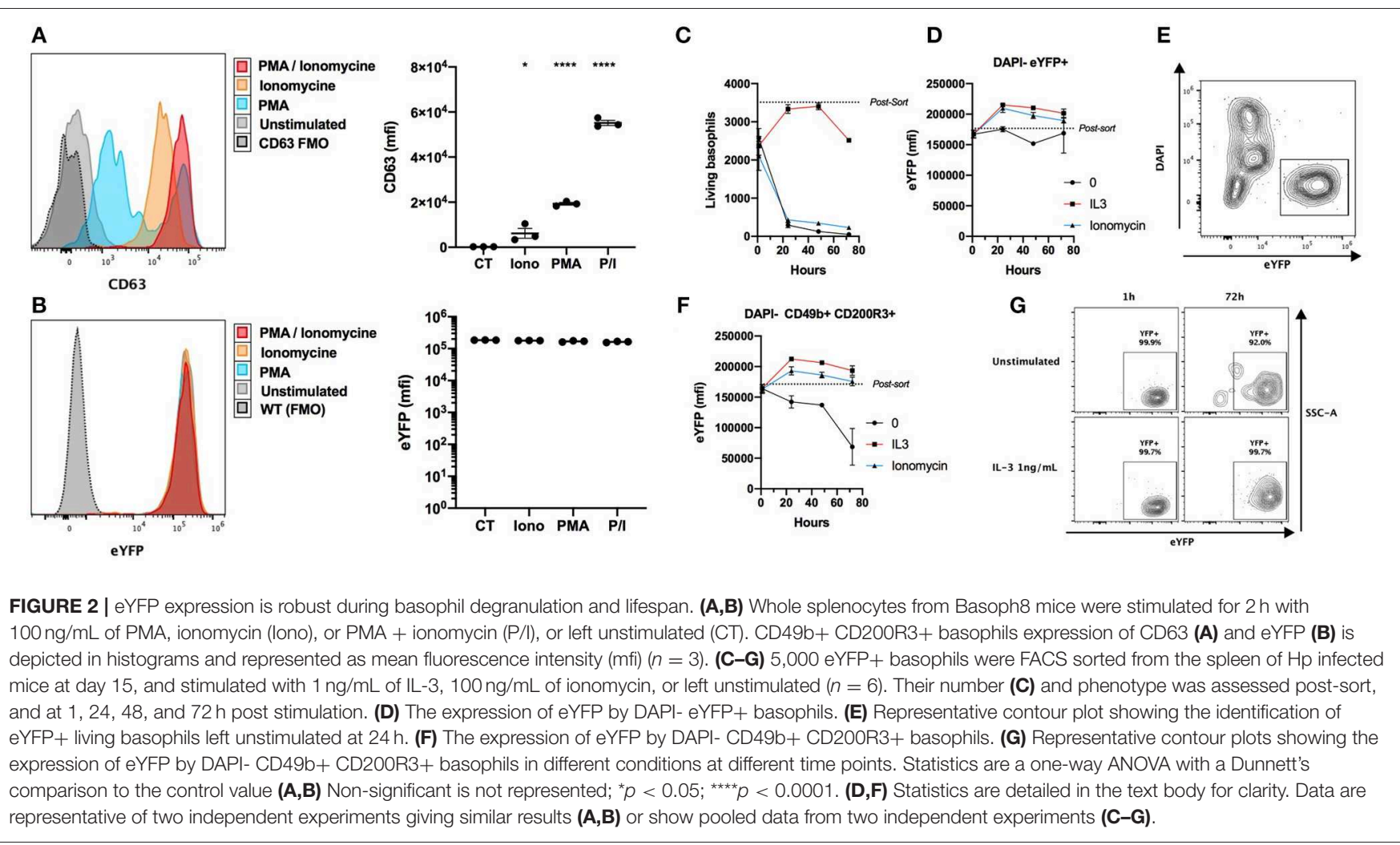

and/or CD200R3 (Ba13). However, the incubation of basophils with either of these antibodies can lead to a strong basophil activation and even to anaphylaxis in vivo $(2,8,30)$. In order to control for any basophil staining induced bias, we crossed the Basoph8 mice with the 4C13R strain, which faithfully reports the expression of IL4 and IL13 with AmCyan and dsRed, respectively (19). These mice allow a reliable detection of MCPT8, IL4 and IL13, and the detection of basophils activation by IL-3 without the use of any antibodies (Figure 3A).

Whole splenocytes from $\mathrm{B} 8 \mathrm{x} 4 \mathrm{C} 13 \mathrm{R}$ were stimulated ex vivo with antibody concentrations used commonly for flow cytometry and basophils phenotype was analyzed after $24 \mathrm{~h}$.

2.4G2, MAR1, and Ba13 (Figures 3B-D) all led to a strong activation of basophils, characterized by a strong overexpression of IL4 and IL13. MAR1 and Ba13 also induced an upregulation of the membrane expression of the degranulation marker CD63, which we followed by antibody mediated staining (Figures 3C,D).

Antibody mediated activation during flow cytometry should be inhibited by staining at $4^{\circ} \mathrm{C}$ in media without $\mathrm{Ca}^{2+}$. However, concerns exist about the activation state of basophils after FACS sorting and adoptive transfer in vivo or in coculture experiments in complete medium at $37^{\circ} \mathrm{C}$. Some FACS staining procedures also require steps at $37^{\circ} \mathrm{C}$ (i.e., CXCR5 staining) (33), or in a buffer containing a high calcium content (i.e., Annexin V staining) (29). Basophils from heterozygous B8 mice can be easily FACS sorted based on their strong eYFP expression, without the use of any antibody (Figure 3E), which makes them an ideal model to work with to obtain purified mouse basophils without the bias of an antibody mediated activation.

\section{Basophils Selectively Express the DTR in the B8xiDTR Mouse}

The MCPT8 gene is highly expressed by murine basophils. However, it has also been shown to be expressed by granulocytes macrophages progenitors (GMPs) and to a lesser extent by peritoneal mast cells (13). Very recently Shibata et al. showed that MCPT8 driven Cre mediated recombination was marginal in mature eosinophils and neutrophils and otherwise specific to the basophil lineage (24).

To generate a basophil specific conditional knockout mouse expressing a useful reporter gene, we bred the B8xiDTR mice. The iDTR mouse allows the expression of the simian Diphteria Toxin receptor (DTR) gene, or Heparin Binding Epidermal Growth Factor $(H B E G F)$, after the excision of a floxed STOP codon by Cre recombinase activity, and can be used to generate conditional knockout mice (25).

We quantified the expression of $H B E G F$ in the main immune cell populations of the B8xiDTR mice after FACS sorting. Basophils were the only cell type analyzed that specifically expressed $H B E G F$ in the spleen. However, we detected a low but significant expression of $H B E G F$ on FACS sorted $\mathrm{cKit}^{+}$ CD200R3 ${ }^{+}$peritoneal mast cells (Figure 4A), which amounted to $22.2 \pm 5.9 \%$ of basophils' HBEGF expression. It should be noted that a minimal expression of $H B E G F$ was also detected in eosinophils, neutrophils, macrophages, $\mathrm{B}$ cells and $\mathrm{T}$ cells of the 


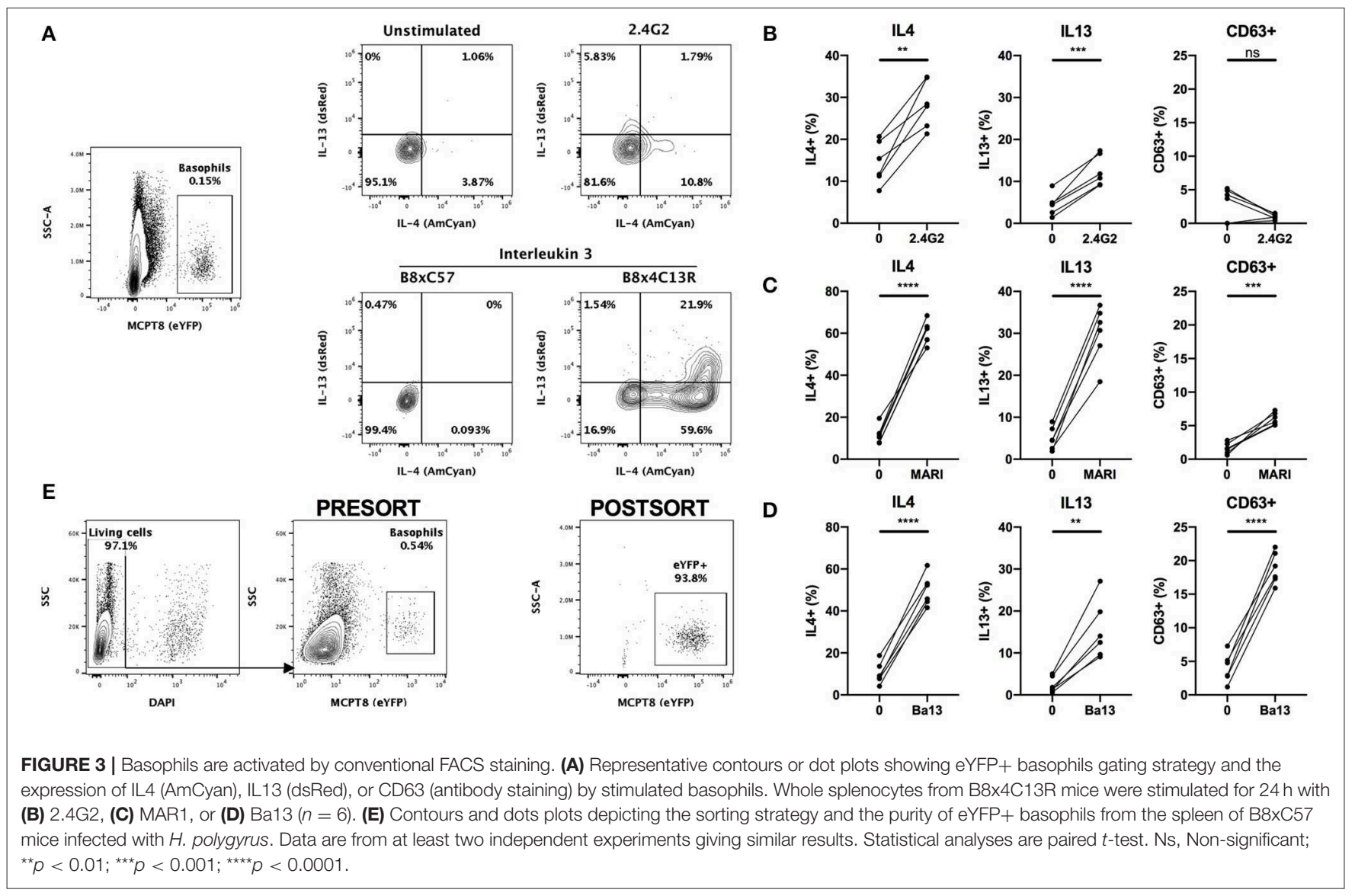

B8xiDTR lineage, but levels were $<10 \%$ of the expression levels in basophils (Figure 4B). These results indicate that MCPT8 driven Cre mediated recombination can occur at minimal but significant levels in cells other than basophils in the Basoph8 mice progeny. They suggest that less than $\sim 25 \%$ of peritoneal mast cells and $10 \%$ of others cells can express the DTR in the B8xiDTR model.

\section{The B8xiDTR Mice Allows for a Relatively Specific Depletion of Basophils ex vivo}

We studied the efficiency and the specificity of DT mediated depletion of basophils from the B8xiDTR mice ex vivo. Bone marrow (BM) from B8xiDTR and $\mathrm{B} 8 \mathrm{xC} 57$ mice was stimulated with increasing doses of DT and immune cell populations were quantified by flow cytometry. Only basophils from B8xiDTR were depleted among all the cell types analyzed including neutrophils, $\mathrm{Ly}_{6 \mathrm{C}}{ }^{+}$and Ly6C $\mathrm{C}^{-}$monocytes, B cells, eosinophils, $\mathrm{CD}^{+}{ }^{+}$and $\mathrm{CD}^{+}{ }^{+}$T cells, and plasmacytoid dendritic cells (pDCs) (Figure 5A). Similarly, only basophils were depleted among the main immune subsets from whole splenocytes (Figure 5B). As the peritoneal cavity contains a unique immune compartment with highly specialized cells, including pMCs, but very few basophils, we also stimulated whole peritoneal lavage cells from B8xiDTR mice ex vivo with the highest dose of DT shown to deplete both BM and spleen basophils. Neither peritoneal B cells, macrophages nor mast cells were depleted by this high dose of DT (Figure 5C), indicating that DT selectively depletes basophils in the B8xiDTR mouse ex vivo. Importantly, we checked that basophils defined not only as eYFP ${ }^{+}$but also as CD $45^{\text {low }}$ FceRIa ${ }^{+}$cells were depleted by a high dose of DT in B8xiDTR mice spleens (Supplementary Figure 2), and that no cell subset was depleted in the B8xC57 background controls (Supplementary Figure 3).

\section{The B8xiDTR Mice Allows a Relatively Specific Depletion of Basophils in vivo}

Our ex vivo experiments supported that basophils were selectively depleted by DT from the spleen and the BM of B8xiDTR mice, but we wanted to rule out whether off target effects could arise in vivo following several days of depletion. One injection of DT was never sufficient to significantly deplete basophils the following day in the blood or the spleen even at a dose of $100 \mathrm{ng} / \mathrm{g}$ (data not shown). Repeated daily DT injections (20 ng/g i.p.) allowed for a depletion of peripheral blood basophils with no significant effect on any other cells analyzed (neutrophils, B cells, $\mathrm{CD}^{+}$and $\mathrm{CD}^{+}{ }^{+} \mathrm{T}$ cells, $\mathrm{Ly}_{6 \mathrm{C}^{+}}$ or Ly6C $\mathrm{C}^{-}$monocytes, eosinophils or NK cells) during the first week (Figure 6A). The depletion was significant after 2 days and plateaued at day $3(87.6 \pm 1.6 \%)$ for at least 2 days after the last DT injection. The recovery was variable between experiments and mice (beginning from day 3 to 5 post DT 

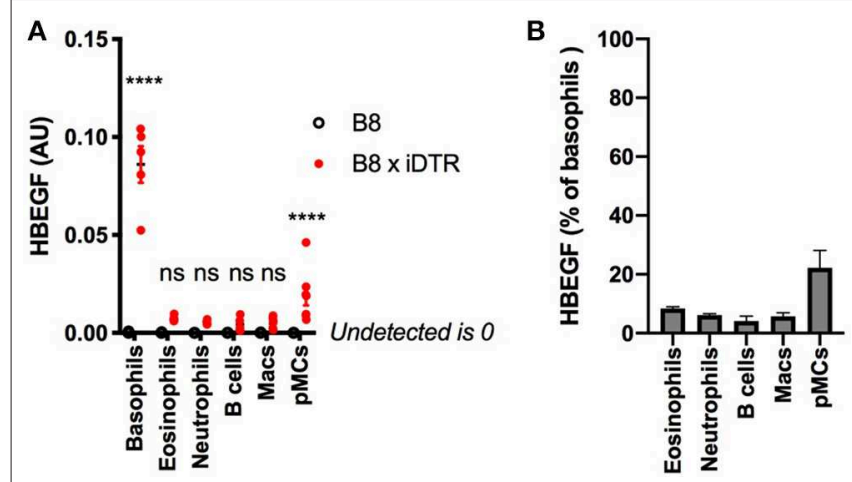

FIGURE 4 | Analysis of the specificity of DTR expression in B8xiDTR mice. (A) Expression of HBEGF was analyzed by RTqPCR on FACS sorted eYFP ${ }^{+}$ Basophils, CD11b ${ }^{+}$SiglecF ${ }^{+}$SSChi Eosinophils, CD11b ${ }^{+}$Ly6G $^{+}$Neutrophils from the spleens and CD11b+ CD64 ${ }^{+}$Macrophages, CD19 ${ }^{+} \mathrm{B}$ cells or

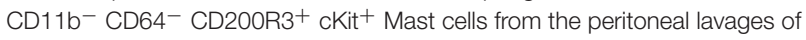
B8xiDTR or B8xC57 mice infected for 15 days with $\mathrm{Hp}$ (for the spleens) or not (peritoneum), and normalized to their expression of GAPDH $(n=5)$.

(B) Illustration of the expression of HBEGF by B8xiDTR immune cells from

(A) as a \% of the mean expression by B8xiDTR basophils. Data are representative of at least two independent experiments giving similar results. Statistical analyses are a two-way ANOVA with a Sidak post-test comparing the different genotypes for each immune cell population. Ns, Non significant; ${ }^{\star \star \star \star} p<0.0001$.

injection). After 7 daily DT injections we noticed a significant drop in $\mathrm{Ly} 6 \mathrm{C}^{+}$monocyte numbers that did not recover after stopping the DT treatment (Figure 6A). Variable signs of sickness (behavior, cold to the touch) occurred after long term daily DT treatment ( $>10$ days) in roughly half of the mice (data not shown). We hypothesized that these symptoms could be due to an anaphylactic reaction to repeated DT injections. Consequently, we decided to limit the depletion to 7 daily DT injections in the B8xiDTR model.

Basophils were also depleted in the spleen after 3 or 7 days of daily DT injections $(86.4 \pm 5.02 \%)$ while neither B cells, $\mathrm{CD}^{+}$or $\mathrm{CD}^{+} \mathrm{T}$ cells, neutrophils, inflammatory monocytes, eosinophils nor DCs were depleted (Figure 6B). Similarly, DT injections did not change the numbers of peritoneal mast cells or B cells, or mast cells from the ear skin, but we noticed a significant increase in the proportion of peritoneal macrophages after 7 days of DT injections when compared with PBS injections (Figure 6C).

The injection of DT induced the depletion of BM basophils to a lesser extent $(60.4 \pm 2.1 \%)$ than in other sites, as previously reported in the Basoph8xDTa model (21). El Hachem et al. showed that the injection of a strong dose of DT could induce a depletion of Granulocytes Macrophages Progenitors (GMPs), which led to a depletion of mature eosinophils and neutrophils in the MCPT8-DTR model (13). Here, unexpectedly, the repeated injection of DT induced an increase in the number of the GMPs in the BM, but no significant differences in the numbers of Common Myeloid Progenitor (CMPs) or Megakaryocyte Erythroids Progenitor (MEPs) (Figure 6D). Interestingly, we noticed a minor fraction of GMPs that expressed the eYFP reporter and these were not depleted by DT injections, suggesting

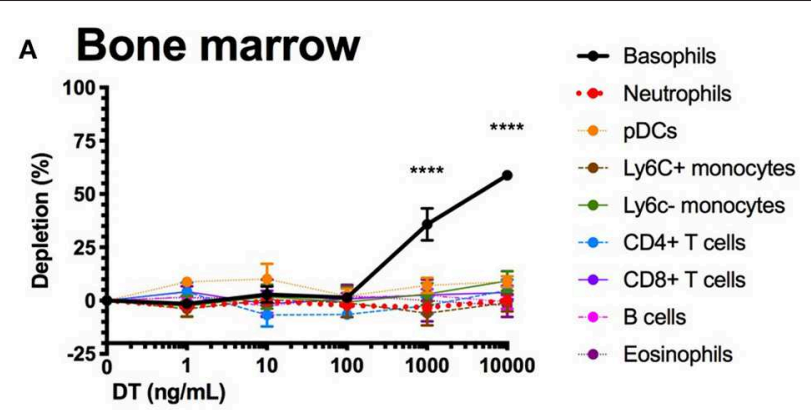

B Spleen

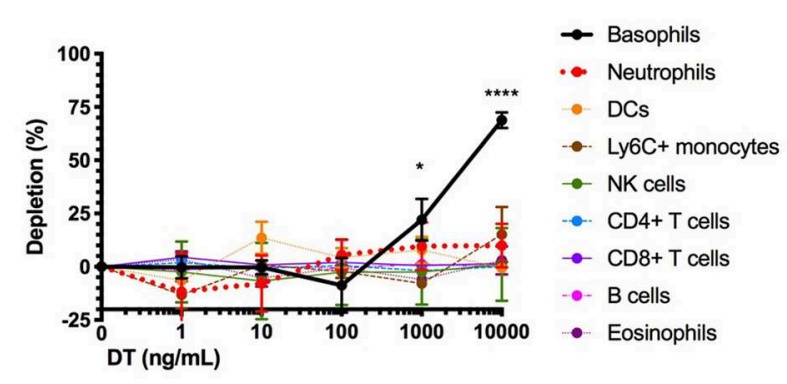

\section{c Peritoneal cavity}
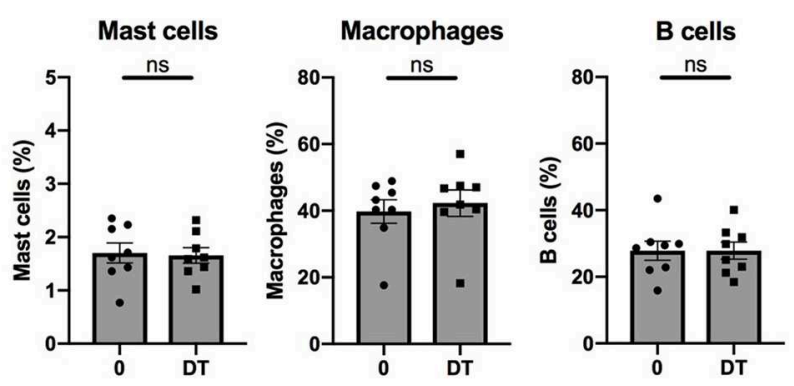

FIGURE 5 | Basophils from B8xiDTR mice can be specifically depleted ex vivo. (A) eYFP ${ }^{+}$Basophils, Ly6G $^{-}$SiglecF $^{+}$SSC $^{\text {hi }}$ eosinophils, B220 ${ }^{+}$ $\mathrm{MHCll}^{+} \mathrm{B}$ cells, CD3 ${ }^{-} \mathrm{NK} 1.1^{+} \mathrm{NK}$ cells, CD11b ${ }^{+} \mathrm{Ly} 6 \mathrm{G}^{+}$Neutrophils, CD3 ${ }^{-}$ SiglecF ${ }^{-} \mathrm{Ly}_{6 G^{-}} \mathrm{CD}_{11 \mathrm{~b}}{ }^{+} \mathrm{CX} 3 \mathrm{CR} 1^{+} \mathrm{Ly}_{6 \mathrm{C}^{-}}$or $\mathrm{Ly}_{6 \mathrm{C}^{+}}$monocytes, CD3 ${ }^{+}$

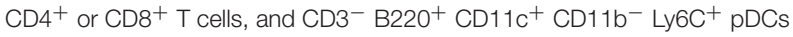
numbers were quantified by flow cytometry after stimulation for $24 \mathrm{~h}$ of whole femur bone marrow of B8xiDTR or B8xC57 mice with increasing doses of DT $(n=6)$. (B) eYFP+ Basophils, SiglecF+ $\mathrm{SSC}^{\text {hi }}$ eosinophils, $\mathrm{B}^{+2} \mathrm{O}^{+} \mathrm{MHCl}^{+} \mathrm{B}$ cells, CD3-NK1.1+ NK cells, CD11b+ Ly6G $^{+}$Neutrophils, CD3 - SiglecF $^{-}$ $\mathrm{Ly}_{6 \mathrm{G}^{-}} \mathrm{CD}_{11 \mathrm{~b}}{ }^{+} \mathrm{Ly}_{6 \mathrm{C}^{+}}$monocytes, $\mathrm{CD}^{+} \mathrm{CD}^{+}{ }^{+}$or $\mathrm{CD}^{+}{ }^{+} \mathrm{T}$ cells, and $\mathrm{CD}^{-} \mathrm{B220}^{-} \mathrm{CD}^{-11 \mathrm{C}^{+}} \mathrm{MHCll}^{\text {hi }}$ DCs numbers were quantified by flow cytometry after stimulation for $24 \mathrm{~h}$ of whole splenocytes of B8xiDTR or B8xC57 mice with increasing doses of DT $(n=6)$. (C) Peritoneal lavages of B8xiDTR mice were stimulated or with $10 \mu \mathrm{g} / \mathrm{mL}$ of DT for $24 \mathrm{~h}$ and the number of CD64+ Macrophages, CD19+ $\mathrm{B}$ cells and CD200R3 ${ }^{+} \mathrm{cKit}^{+}$Mast cells were quantified by flow cytometry. Data are from two independent experiments giving similar results $(n=8)$. (A,B) Data has been normalized on the unstimulated condition and represents only the B8xiDTR genotype. Statistical analyses are $(\mathbf{A}, \mathbf{B})$ a two-way ANOVA with a Sidak post-test comparing matched stimulated with unstimulated conditions or (C) a paired $t$-test. Ns, Non significant; ${ }^{\star} p<0.05 ;{ }^{* \star \star \star} p<0.0001$.

that if the eYFP-Cre recombinase transgene is expressed at the GMP stage in the B8xiDTR mouse, its expression did not allow a significant recombination, expression of the DTR, and DT 


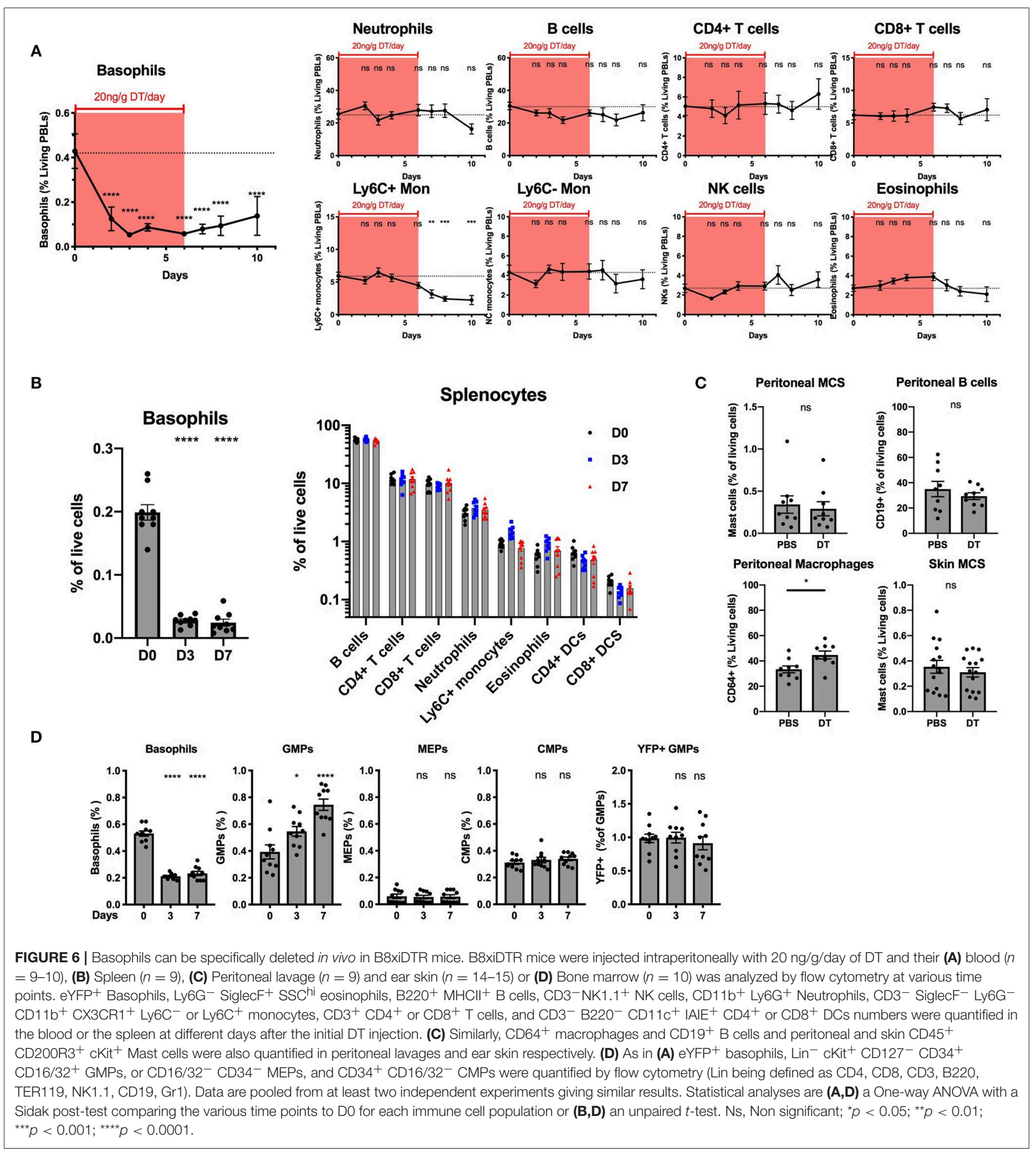

mediated depletion (Figure 6D). Overall, the B8xiDTR mice allowed a conditional depletion of basophils for 7 consecutive days from 2 days after the first injection to 2 days after the last injection, but this depletion seemed relatively specific only during the first week of DT injection.

\section{The B8xiDTR Mice Enables the Depletion of Basophils in the Skin During Allergic Inflammation}

Basophils infiltrate many tissues upon activation, including the gut, the liver, the lungs and the skin during helminth infection 


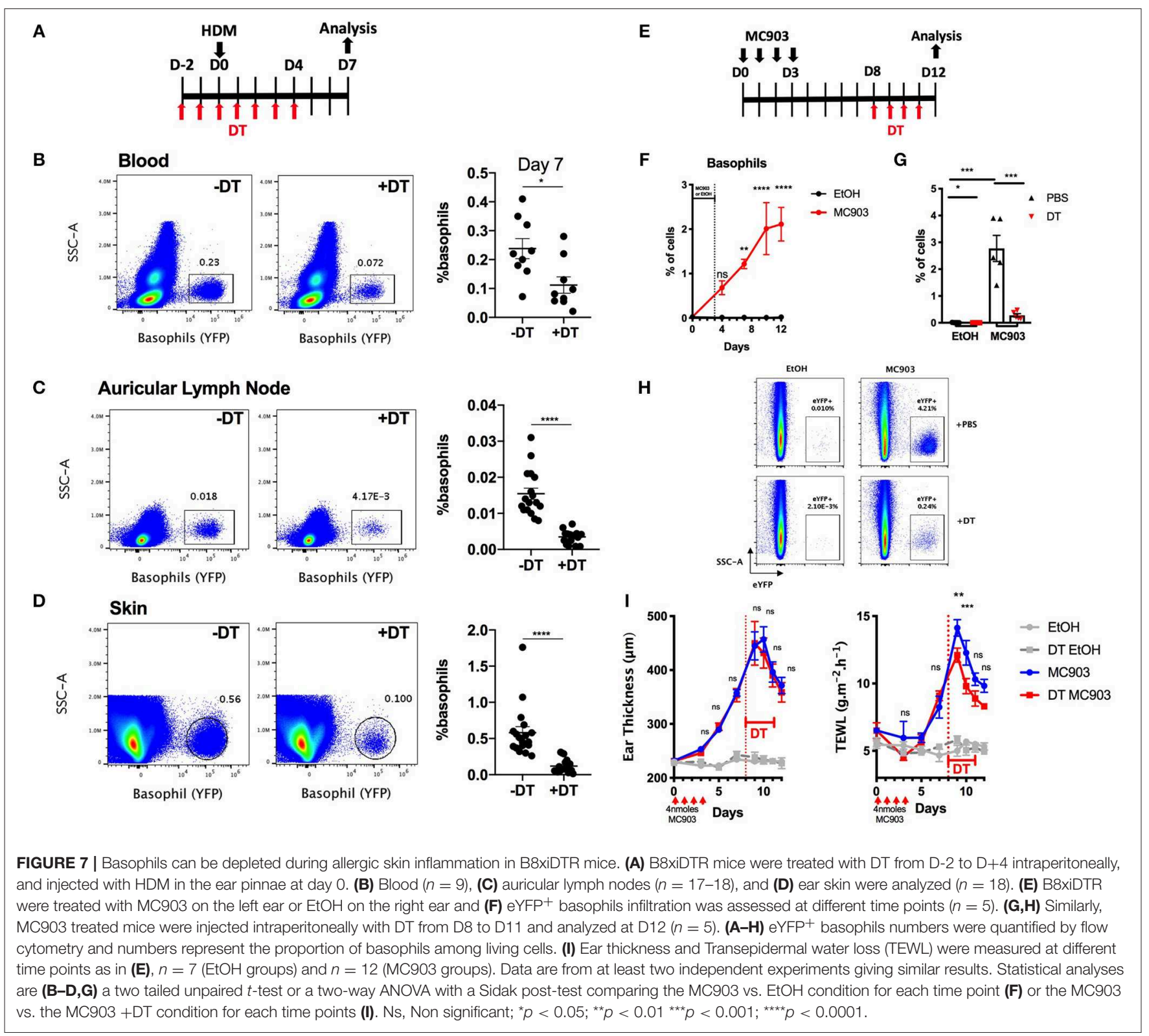

(34). DT mediated cell depletion in the skin is usually more difficult to achieve as this organ is less vascularized than the lung, the spleen or the liver (35). We evaluated the efficiency of basophils depletion in the skin in the B8xiDTR model in a model of HDM induced skin allergy (28). The intraperitoneal injection of DT from day -2 to day 4 allowed an efficient depletion of basophils up to day 7 in both the ear skin and its draining lymph nodes, while the number of peripheral blood basophils was beginning to return to baseline at day 7, 3 days after the last DT injection (Figures 6A, 7A-D).

Basophils also accumulate in the skin during a model of MC903 induced atopic dermatitis, their numbers plateauing at day 9-10 (Figures 7E,F). In order to assess if we could efficiently deplete basophils in the skin tissue after they began to infiltrate it, MC903 treated B8xiDTR mice were treated with DT from Day 9 to 12 and the ear skin was analyzed by flow cytometry at day 13 post MC903 treatment. Basophils were efficiently depleted from the skin tissue (Figures 7E,G,H). Surprisingly, basophil depletion at this late time point was not associated with any significant changes in the development of ear inflammation, as measured by the ear thickness. However, we noticed that basophils depletion induced a transient decrease in the development of the transepidermal water loss (TEWL), a measure of the barrier function of the epidermis (Figure 7I) (36).

Helminth infection induces a potent basophilia in mice. To assess the depletion capabilities of the B8xiDTR model during helminth infection, we injected DT dayly for 10 days, starting 3 days prior to $N$. brasiliensis $(\mathrm{Nb})$ infection of B8xiDTR or B8xC57 mice. Peripheral blood basophils numbers were consistently depleted by more than $80 \%$ compared to DT treated B8xC57BL/6 
or PBS treated B8xiDTR mice during the course of the infection (Supplementary Figure 4).

In conclusion, the B8xiDTR model allows identification and sorting of basophils by flow cytometry in an unbiased fashion and their selective depletion in various tissues at steady state and during allergic or Type 2 inflammation.

\section{MATERIALS AND METHODS}

\section{Mice and Treatments}

C57BL/6J, Basoph8 (21), iDTR (25) (Jackson Laboratories), and the 4C13R (19) mice were bred and housed in specific pathogen-free conditions at the Malaghan Institute of Medical Research Biomedical Research Unit. Basoph8 mice progeny were kept as heterozygous and sufficient for MCPT8, a protease showing potent pro-inflammatory properties during skin allergic inflammation (37). All experimental protocols were approved by the Victoria University of Wellington Animal Ethics Committee (Permit 24432) and performed according to Institutional guidelines. MC903 (Calcipotriol, Cayman Chemicals) was diluted in ethanol and 4nmoles per day were applied topically for 4 days in $20 \mu \mathrm{L}$. House Dust Mite (HDM, Greer) immunization was via intradermal injection of $200 \mu \mathrm{g}$ into the ear pinnae as previously described on mice sedated with an i.p. injection of ketamine/xylazine (28). Diphteria toxin (Cayman Chemical) was injected intraperitoneally at $20 \mathrm{ng} / \mathrm{g} / \mathrm{day}$, unless specified. $N$. brasiliensis $(\mathrm{Nb})$ and $H$. polygyrus $(\mathrm{Hp})$ were maintained via passage through Lewis rats and C57BL/6 mice, respectively, and infective L3 larvae prepared from fecal cultures, as previously described (38). For every infection experiment $200 \mathrm{Hp}$ L3 were given by oral gavage or $550 \mathrm{Nb} \mathrm{L3}$ were injected in the scruff in $200 \mu$ l phosphate-buffered saline (PBS).

\section{Tissues Digestion and Stimulations}

Blood was collected by cheek bleeding or intracardial puncture. Spleens were smashed through a $70 \mu \mathrm{m}$ nylon mesh filter (Becton Dickinson). Primary BM cells were harvested by flushing femurs' content using IMDM (Gibco). Red blood cells were lysed in an ammonium chloride buffer, and peritoneal lavages were harvested, as described elsewhere (29). For skin cell preparations, ears were split into the dorsal and ventral layers, minced and digested $30 \mathrm{~min}$ at $37 \mathrm{C}$ in a shaking incubator (150 rpm) in IMDM 5\% FCS (Gibco) containing $2 \mathrm{mg} / \mathrm{mL}$ of collagenase IV and $100 \mu \mathrm{g} / \mathrm{mL}$ DNAse I (both Sigma). Digestion was stopped by adding $5 \mathrm{mM}$ EDTA and a single cell suspension obtained by smashing the remaining tissue through a $70 \mu \mathrm{m}$ nylon mesh filter (BD). Cells were cultured ex vivo in DMEM 20\% FCS + Non-essential amino acid + Sodium pyruvate (Gibco) at $37 \mathrm{C}+5 \% \mathrm{CO}_{2}$. Peritoneal lavages incubation with DT was done in the presence of $10 \mathrm{ng} / \mathrm{mL}$ of Stem cell factor (SCF) (Peprotech). Whole splenocytes were stimulated with IL3 (Peprotech) or sterilefiltered FcBlock (2.4G2, Becton Dickinson, $10 \mu \mathrm{g} / \mathrm{mL}$ ), MAR1 $(0.5 \mu \mathrm{g} / \mathrm{mL})$ or Ba13-APC (Biolegend, $1 \mu \mathrm{g} / \mathrm{mL})$, Phorbolmyristate acetate (PMA, $100 \mathrm{ng} / \mathrm{mL})$, ionomycin $(100 \mathrm{ng} / \mathrm{mL}$ ) or both (Thermofischer) for the indicated times in culture medium.

\section{Ear Measurements}

Ear thickness was measured on sedated mice with the help of a digital caliper. Transepidermal water loss (TEWL) was similarly measured on both ears of sedated mice using the DERMALAB $^{\text {TM }}$ TEWL probe (Cortex Technology Denmark) at room temperature, as previously described (36).

\section{Flow Cytometry}

Unless specified, single cell suspensions used for flow cytometry were first blocked for $15 \mathrm{~min}$ at $4^{\circ} \mathrm{C}$ in FACS buffer (PBS $1 \%$ bovine serum albumin $0.05 \% \mathrm{NaN}_{3}$, Sigma) containing $0.5 \%$ of $2.4 \mathrm{G} 2$ hybridoma supernatant. Cells were then stained in FACS buffer for $20 \mathrm{~min}$ at $4^{\circ} \mathrm{C}$ with an optimized concentration of fluorophore-conjugated antibodies. Antibodies used for flow cytometry or stimulations were directed against the following mouse antigens: CD45 (30F11), CD11b (M1/70), IgE (R3572), B220 (RA3-6B2), CD117 (2B8), CD11c (HL3), CD16/32 (2.4G2), CD3 (145-2C11), CD4 (RM4-5), CD8a (53-6.7), CD19 (ID3), Ly6G (1A8), NK1.1 (PK136), SiglecF (E50-2440) from Becton Dickinson, CD200R3 (Ba13), Ly6C (HK1.4), CD34 (MEC14.7), CD63 (NVG-2), MHCII (M5/114.15.2), CD64 (X545/7.1), CX3CR1 (SA011F11), Sca1 (D7), Gr1 (RB6-8C5), TER119 (TER119) from Biolegend, FceRI $\alpha$ (MAR1), CD49b (DX5) from eBiosciences.

Doublets and non-viable cells were identified and always excluded using DAPI or LIVE/DEAD staining (Molecular Probes). Compensation was performed using OneComp eBeads (Invitrogen) as single stained positive controls or dsRed or Basoph8 splenocytes or bone marrow cells and fluorescence minus one (FMO) controls were used to set background expression when needed. Basoph8 cells were used to set the background fluorescence level for Basoph8x4C13R cells as FMO. Flow cytometry was performed on an Aurora spectral cytometer (Cytek) or a custom BD LSR Fortessa ${ }^{\mathrm{TM}}$ SORP flow cytometer (6 lasers: 355, 405, 445, 488, 532, $640 \mathrm{~nm}$ ). FACS sorting was carried out on a BD Influx ${ }^{\mathrm{TM}}$ (both from Becton Dickinson). Analyses were conducted using FlowJo vX (Tree Star).

\section{Molecular Biology}

Ten thousand FACS sorted cells were collected in RNA lysis buffer and RNA was extracted using a Quick-RNA kit (Zymo research). cDNA was synthetized using the High Capacity RNAto-cDNA kit (Applied Biosystems). RT-qPCR was performed using Taqman master mix as a duplex with the following Taqman probes: murine GAPDH-Vic (Mm00484668_ml) and human HBEGF-Fam (Hs00181813_m1) using a QuantStudio 7 (Applied Biosystems) and following the manufacturer's guidelines. It is worth underlining that the iDTR mice allow the expression of the simian DTR (HBEGF) after Cre-mediated recombination (25). Primers for the human sequence were used here, which shares 98\% homology with the iDTR simian cloned version. Transcript levels are expressed as the ratio of $2^{-\Delta \mathrm{CT}}$ to GAPDH.

\section{Statistical Analysis}

Statistical analyses were performed using Prism 8.0 (GraphPad). In all cases, a two-tailed $p$-value $<0.05$ was considered as threshold for significance. Statistical tests are indicated in figure's 
legends. Individual dots and/or mean and SEM are shown in all graphs.

\section{DISCUSSION}

Basophils are potent circulating granulocytes that have been associated with the development of allergic or autoimmune diseases and protection against helminths. While their nonredundant role in an acquired resistance against tick infestation has been well established, their contribution to primary or secondary helminth immunity seems to be species dependent (12, $21,26,39-41)$. The dysregulation of basophils is also increasingly being recognized as important for the development of allergic or autoimmune diseases such as atopic dermatitis, food allergy, eosinophilic esophagitis, systemic lupus erythematosus, chronic urticaria and ulcerative colitis, among others (29, 40, 42-46).

Research on mouse basophils has been impeded for a long time by their relative rarity and an elusive phenotype during chronic inflammation. Very recently, MAR1, the antibody commonly used to identify and deplete basophils as Fc\&RI $\alpha^{+}$ cells, was shown to also bind FcgRI and FcgRIV, allowing their detection by flow cytometry on monocytes and macrophages subsets (9). Similarly, the discovery of $\mathrm{CD}_{4} 9 \mathrm{~b}^{+} \mathrm{CD} 200 \mathrm{R} 3^{+}$ tolerogenic DCs is challenging the strategies of identification of basophils relying on these markers (7). Furthermore, we show that the expression of FceRI $\alpha$ and CD200R3 on basophils during helminth infection and allergic inflammation is extremely variable (Figure 1). We also show that basophils express the monocytic marker Ly6C upon activation, which suggest that $\mathrm{Ly}_{6 \mathrm{C}} \mathrm{C}^{+}$basophils could be mistaken for Ly6C ${ }^{+}$Fс\&RI $\alpha^{+}$ monocytic cells.

The identification of basophils by antibodies targeting FceRI $\alpha$, CD200R3, or CD16/32 can also harm their functional study as their binding directly induces basophils degranulation and/ or cytokine expression both ex vivo (Figure 3 ) and in vivo, as previously reported with different readouts $(2,8,30)$. This casts a doubt on the activation effects of the sorting process in some adoptive transfer experiments using these antibodies $(11,45,46)$. Of note, some other strategies have also been used, such as sorting basophils as CD49b + CD $45^{\text {lo }}$ cells (47).

Conversely, the high expression of eYFP in basophils from the Basoph8 mice is specific (23), expressed for at least $72 \mathrm{~h}$, and can be detected even after basophils degranulation or in autofluorescent tissues such as the skin (Figures 1, 2). Basophils can also be FACS sorted by using this reporter expression, which would allow to overcome any potential antibody staining mediated activation during the purification process (Figure 3). These advantages greatly facilitate a reliable identification, isolation by FACS sorting, and study of basophils in vivo.

The expression of MCPT8 is specific to basophils among mature cells. However, it is still transiently expressed at the progenitor stage, to a level sufficient to allow their depletion by a high dose of DT in the MCPT8-DTR model (13). This raised concerns about the specificity of MCPT8 Cre-mediated recombination in the B8xiDTR model. To understand the occurrence of these genetic events in other immune cells we quantified the expression of HBEGF in various immune cells from the B8xiDTR mice. Indeed, we could detect low levels of expression of HBEGF, especially in peritoneal mast cells, eosinophils and neutrophils of these mice.

HBEGF is expressed constitutively under the control of Rosa26 in the iDTR model (25). As such, its expression levels should be similar in all cells having experienced a Cre mediated recombination event. This allowed us to estimate that $\sim 20 \%$ of peritoneal mast cells, and $<10 \%$ of eosinophils or neutrophils are affected by Cre mediated recombination events in the B8xiDTR mice (Figure 4). These numbers are consistent with what has been observed very recently by Shibata and al (24). Importantly, this "non-specificity" did not lead to a significant detectable depletion of any immune cell analyzed ex vivo or in vivo in the B8xiDTR mice, which support the idea that Cre-mediated recombination is predominantly occurring solely in basophils in heterozygous Basoph8 mice (Figures 5, 6).

After $24 \mathrm{~h}$ of stimulation ex vivo, only high doses of DT ( 1 to $10 \mu \mathrm{g} / \mathrm{mL}$ ) were able to significantly deplete basophils (Figure 5). In vivo, preliminary studies revealed that even high doses of DT, up to $100 \mathrm{ng} / \mathrm{g}$, were not able to deplete peripheral blood basophils after $24 \mathrm{~h}$. Indeed, the depletion was effective only after repeated infections of DT in this model, being optimal only at day 2 after the first injection. This depletion of basophils seems less sensitive to DT than in the MCPT8-DTR model, where one injection of DT sufficed to deplete basophils from day 2-5 (12). We did not observe any significant depletion of immune cells or progenitors over a 7 days period, as it was observed on the MCPT8-DTR mice (13). These differences likely reflect a difference in the effective expression of the DTR at the basophil's membrane between both models. Indeed, the transcription of DTR-eGFP expression is driven by the MCPT8 promoter in the MCPT8-DTR mice, and its transcription controlled by an IRES (12), but the DTR expression is driven by Rosa26 in the B8xiDTR mice and its mRNA does not contain an IRES (25).

Over a 7 days period of depletion, we did observe some variation in the numbers of some immune cells such as an increase in peritoneal macrophages numbers, and an increase in the proportion of GMPs in the BM (Figure 6). These increases could be due to a complex interaction of basophils with others immune cells, their depletion freeing tissue or cytokine niches, and have to be carefully considered as such. A longterm depletion of basophils using the B8xiDTR did not seem achievable per se: after 7 days of DT injections, we noticed a 2 -fold decrease in peripheral blood inflammatory monocytes, which can have a significant impact on the development of immune responses. More concerning, after more than 10 days of daily DT injections half of the mice began to look sick and cold, which was likely due to an anaphylactic response to the frequent DT injections. We do not recommend depleting basophils using the B8xiDTR mice for more than six consecutive days due to these effects.

Basophils depletion was efficient in two models of skin allergic inflammation such as after an intradermal injection of HDM or in an MC903 induced atopic dermatitis model. Beginning at 2 days before HDM injection, DT injection was sufficient to deplete basophils in the ear skin and its draining lymph nodes for at least 9 days. It is worth underlining that at this time point, 3 days after the last DT injection, basophil numbers were beginning to get back to baseline in the 
peripheral blood. A concern of DT mediated cell depletion is the bioavailability of DT in tissues, especially those that are not highly vascularized (35).

MC903 topical application on the skin induce a potent recruitment of basophils, which peaks at day 9 in our model. The intraperitoneal injection of DT from day 8 to 11 led to a potent depletion of basophils in the ear skin, suggesting that tissue infiltrating basophils were depleted by this regimen in the B8xiDTR mice (Figure 7). This enabled us to show that basophils were involved in the development of the barrier dysfunction, but not ear thickening, at the late time points of this model of atopic dermatitis. This contrasts results obtained from similar models suggesting pro-inflammatory or anti-inflammatory effects of basophils $(45,48,49)$, and highlights that the role of basophils in the atopic skin deserves a more thorough investigation. The delivery of DT by different routes in the B8xiDTR model, such as the intranasal or intradermal ones, seems worth investigating as to specifically target basophils locally and transiently.

In conclusion, the Basoph 8 mice can be used to easily identify basophils and FACS sort them without the biases of relying on a stringent phenotype, or of using activating antibodies, respectively. It is also an interesting tool to breed new strains such as the B8xiDTR to deplete basophils specifically over a short period of time in inflammatory conditions. We argue that this is a commercially available tool that can greatly facilitate the study of murine basophils in vivo.

\section{DATA AVAILABILITY}

The datasets generated for this study are available on request to the corresponding author.

\section{REFERENCES}

1. Karasuyama H, Miyake K, Yoshikawa S, Yamanishi Y. Multifaceted roles of basophils in health and disease. J Allergy Clin Immunol. (2018) 142:370-80. doi: 10.1016/j.jaci.2017.10.042

2. Kojima T, Obata K, Mukai K, Sato S, Takai T, Minegishi Y, et al. Mast cells and basophils are selectively activated in vitro and in vivo through CD200R3 in an IgE-independent manner. J Immunol. (2007) 179:7093-100. doi: 10.4049/jimmunol.179.10.7093

3. Ohnmacht C, Voehringer D. Basophil effector function and homeostasis during helminth infection. Blood. (2009) 113:2816-25. doi: 10.1182/blood-2008-05-154773

4. Hammad H, Plantinga M, Deswarte K, Pouliot P, Willart MA, Kool M, et al. Inflammatory dendritic cells-not basophils-are necessary and sufficient for induction of Th2 immunity to inhaled house dust mite allergen. J Exp Med. (2010) 207:2097-111. doi: 10.1084/jem.20101563

5. Obata K, Mukai K, Tsujimura Y, Ishiwata K, Kawano Y, Minegishi Y, et al. Basophils are essential initiators of a novel type of chronic allergic inflammation. Blood. (2007) 110:913-20. doi: 10.1182/blood-2007-01-068718

6. Nakamura T, Fukaya T, Uto T, Takagi H, Arimura K, Tono T, et al. Selective depletion of basophils ameliorates immunoglobulin E-mediated anaphylaxis. Biochem Biophys Rep. (2017) 9:29-35. doi: 10.1016/j.bbrep.2016.11.001

7. Sato K, Eizumi K, Fukaya T, Fujita S, Sato Y, Takagi H, et al. Naturally occurring regulatory dendritic cells regulate murine cutaneous chronic graft-versus-host disease. Blood. (2009) 113:4780-9. doi: 10.1182/blood-2008-10-183145

\section{ETHICS STATEMENT}

All experimental protocols were approved by the Victoria University of Wellington Animal Ethics Committee (Permit 23910) and performed according to Institutional guidelines.

\section{AUTHOR CONTRIBUTIONS}

$\mathrm{CP}, \mathrm{PM}, \mathrm{MP}, \mathrm{KN}, \mathrm{BY}, \mathrm{JC}, \mathrm{SC}, \mathrm{KF}, \mathrm{MC}$, and $\mathrm{GL}$ designed and/or conducted experiments. CP and GL wrote the manuscript. GL supervised the project. All authors provided feedback on the manuscript.

\section{FUNDING}

This research was funded by an independent research organization grant from the Health Research Council of New Zealand and by the Marjorie Barclay Trust.

\section{ACKNOWLEDGMENTS}

The authors wish to thank the expert support of the Malaghan Institute of Medical Research Hugh Green Cytometry Core, Research Information Technologies, and Biomedical Research Unit staff.

\section{SUPPLEMENTARY MATERIAL}

The Supplementary Material for this article can be found online at: https://www.frontiersin.org/articles/10.3389/fimmu. 2019.02143/full\#supplementary-material

8. Hübner MP, Larson D, Torrero MN, Mueller E, Shi Y, Killoran KE, et al. Anti-FceR1 antibody injections activate basophils and mast cells and delay Type 1 diabetes onset in NOD mice. Clin Immunol. (2011) 141:205-17. doi: 10.1016/j.clim.2011.08.004

9. Tang X-Z, Jung JB, Allen CDC. A case of mistaken identity: the MAR-1 antibody to mouse FceRI $\alpha$ cross-reacts with Fc $\gamma$ RI and Fc $\gamma$ RIV. J Allergy Clin Immunol. (2019) 143:1643-1646.e6. doi: 10.1016/j.jaci.2018.11.045

10. Motomura Y, Morita H, Moro K, Nakae S, Artis D, Endo TA, et al. Basophil-derived interleukin-4 controls the function of natural helper cells, a member of ILC2s, in lung inflammation. Immunity. (2014) 40:758-71. doi: 10.1016/j.immuni.2014.04.013

11. Siracusa MC, Saenz SA, Hill DA, Kim BS, Headley MB, Doering TA, et al. TSLP promotes interleukin-3-independent basophil haematopoiesis and type 2 inflammation. Nature. (2011) 477:229-33. doi: 10.1038/nature10329

12. Wada $T$, Ishiwata $K$, Koseki $H$, Ishikura $T$, Ugajin $T$, Ohnuma $N$, et al. Selective ablation of basophils in mice reveals their nonredundant role in acquired immunity against ticks. J Clin Invest. (2010) 120:2867-75. doi: $10.1172 /$ JCI42680

13. El Hachem C, Hener P, Kirstetter P, Li J, Chan S, Li M. Treatment of MCPT8DTR mice with high- or low-dose diphtheria toxin leads to differential depletion of basophils and granulocyte-macrophage progenitors. Eur J Immunol. (2018) 1:1-13. doi: 10.1002/eji.201747351

14. Sawaguchi M, Tanaka S, Nakatani Y, Harada Y, Mukai K, Matsunaga $\mathrm{Y}$, et al. Role of mast cells and basophils in IgE responses and in allergic airway hyperresponsiveness. J Immunol. (2012) 188:1809-18. doi: $10.4049 /$ jimmunol.1101746 
15. Bao K, Reinhardt RL. The differential expression of IL-4 and IL13 and its impact on type-2 immunity. Cytokine. (2015) 75:25-37. doi: 10.1016/j.cyto.2015.05.008

16. Chen F, Wu W, Millman A, Craft JF, Chen E, Patel N, et al. Neutrophils prime a long-lived effector macrophage phenotype that mediates accelerated helminth expulsion. Nat Immunol. (2014) 15:938-46. doi: 10.1038/ni.2984

17. Sun B, Zhu L, Tao Y, Sun HX, Li Y, Wang P, et al. Characterization and allergic role of IL-33-induced neutrophil polarization. Cell Mol Immunol. (2018) 15:782-93. doi: 10.1038/cmi.2017.163

18. Vantourout P, Hayday A. Six-of-the-best: unique contributions of $\gamma \delta$ T cells to immunology. Nat Rev Immunol. (2013) 13:88-100. doi: 10.1038/nri3384

19. Roediger B, Kyle R, Yip KH, Sumaria N, Guy TV, Kim BS, et al. Cutaneous immunosurveillance and regulation of inflammation by group 2 innate lymphoid cells. Nat Immunol. (2013) 14:564-73. doi: 10.1038/ni.2584

20. Zeng MY, Pham D, Bagaitkar J, Liu J, Otero K, Shan M, et al. An efferocytosisinduced, IL-4-dependent macrophage-iNKT cell circuit suppresses sterile inflammation and is defective in murine CGD. Blood. (2013) 121:3473-83. doi: 10.1182/blood-2012-10-461913

21. Sullivan BM, Liang HE, Bando JK, Wu D, Cheng LE, McKerrow JK, et al. Genetic analysis of basophil function in vivo. Nat Immunol. (2011) 12:527-35. doi: 10.1038/ni.2036

22. Cheng LE, Sullivan BM, Retana LE, Allen CD, Liang HE, Locksley RM. IgEactivated basophils regulate eosinophil tissue entry by modulating endothelial function. J Exp Med. (2015) 212:513-24. doi: 10.1084/jem.20141671

23. Abram CL, Roberge GL, Hu Y, Lowell C. A Comparative analysis of the efficiency and specificity of myeloid-Cre deleting strains using ROSA-EYFP reporter mice. J Immunol Methods. (2014) 408:89-100. doi: 10.1016/j.jim.2014.05.009

24. Shibata S, Miyake K, Tateishi T, Yoshikawa S, Yamanishi Y, Miyazaki Y, et al. Basophils trigger emphysema development in a murine model of COPD through IL-4-mediated generation of MMP-12-producing macrophages. Proc Natl Acad Sci USA. (2018) 115:13057-62. doi: 10.1073/pnas.1813927115

25. Buch T, Heppner FL, Tertilt C, Heinen TJ, Kremer M, Wunderlich FT, et al. A Cre-inducible diphtheria toxin receptor mediates cell lineage ablation after toxin administration. Nat Methods. (2005) 2:419-26. doi: 10.1038/nmeth762

26. Schwartz C, Turqueti-Neves A, Hartmann S, Yu P, Nimmerjahn F, Voehringer D. Basophil-mediated protection against gastrointestinal helminths requires IgE-induced cytokine secretion. Proc Natl Acad Sci USA. (2014) 111:1-9. doi: 10.1073/pnas.141266311

27. Herbst T, Esser J, Prati M, Kulagin M, Stettler R, Zaiss MM, et al. Antibodies and IL-3 support helminth-induced basophil expansion. Proc Natl Acad Sci USA. (2012) 109:14954-9. doi: 10.1073/pnas.1117584109

28. Ochiai S, Jagot F, Kyle RL, Hyde E, White RF, Prout M, et al. Thymic stromal lymphopoietin drives the development of IL-13+ Th2 cells. Proc Natl Acad Sci USA. (2018) 115:1033-8. doi: 10.1073/pnas.1714348115

29. Pellefigues C, Dema B, Lamri Y, Saidoune F, Chavarot N, Lohéac C, et al. Prostaglandin D2 amplifies lupus disease through basophil accumulation in lymphoid organs. Nat Commun. (2018) 9:725. doi: 10.1038/s41467-018-03129-8

30. Iwamoto H, Matsubara T, Nakazato Y, Namba K, Takeda Y. Decreased expression of CD200R3 on mouse basophils as a novel marker for IgG1-mediated anaphylaxis. Immunity Inflamm Dis. (2015) 3:280-8. doi: 10.1002/iid3.67

31. Dvorak AM. A role for vesicles in human basophil secretion. Cell Tissue Res. (1998) 293:1-22. doi: 10.1007/s004410051093

32. Ebo DG, Bridts CH, Mertens CH, Hagendorens MM, Stevens WJ, De Clerck LS. Analyzing histamine release by flow cytometry (HistaFlow): a novel instrument to study the degranulation patterns of basophils. J Immunol Methods. (2012) 375:30-8. doi: 10.1016/j.jim.2011.09.003

33. Elsner RA, Ernst DN, Baumgarth N. Single and coexpression of CXCR4 and CXCR5 identifies CD4 T helper cells in distinct lymph node niches during influenza virus infection. J Virol. (2012) 86:7146. doi: 10.1128/JVI.06904-11

34. Min B, Prout M, Hu-Li J, Zhu J, Jankovic D, Morgan ES, et al. Basophils produce IL-4 and accumulate in tissues after infection with a Th2-inducing parasite. J Exp Med. (2004) 200:507-17. doi: 10.1084/jem.20040590
35. Dahdah A, Gautier G, Attout T, Fiore F, Lebourdais E, Msallam R, et al. Mast cells aggravate sepsis by inhibiting peritoneal macrophage phagocytosis. J Clin Invest. (2014) 124:4577-89. doi: 10.1172/JCI75212

36. Naidoo K, Jagot F, van den Elsen L, Pellefigues C, Jones A, Luo H, et al. Eosinophils determine dermal thickening and water loss in a MC903 model of atopic dermatitis. J Invest. Dermatol. (2018) 138:2606-16. doi: 10.1016/j.jid.2018.06.168

37. Tsutsui H, Yamanishi Y, Ohtsuka H, Sato S, Yoshikawa S, Karasuyama H. The basophil-specific protease mMCP-8 provokes an inflammatory response in the skin with microvascular hyperpermeability and leukocyte infiltration. $J$ Biol Chem. (2017) 292:1061-7. doi: 10.1074/jbc.M116.754648

38. Camberis M, Le Gros G, Urban J Jr. Animal model of Nippostrongylus brasiliensis and Heligmosomoides polygyrus. Curr Protoc Immunol. (2003) 55:19.12.1-27. doi: 10.1002/0471142735.im1912s55

39. van Panhuys N, Prout M, Forbes E, Min B, Paul WE, Le Gros G. Basophils are the major producers of IL-4 during primary helminth infection. J Immunol. (2011) 186:2719-28. doi: 10.4049/jimmunol.1000940

40. Hussain M, Borcard L, Walsh KP, Pena Rodriguez M, Mueller C, Kim $\mathrm{BS}$, et al. Basophil-derived IL-4 promotes epicutaneous antigen sensitization concomitant with the development of food allergy. J Allergy Clin Immunol. (2018) 141:223-234.e5. doi: 10.1016/j.jaci.2017.02.035

41. Inclan-Rico JM, Siracusa MC. First responders: innate immunity to helminths. Trends Parasitol. (2018) 34:861-80. doi: 10.1016/j.pt.2018.08.007

42. Pellefigues $\mathrm{C}$, Charles $\mathrm{N}$. The deleterious role of basophils in systemic lupus erythematosus. Curr Opin Immunol. (2013) 25:704-11. doi: 10.1016/j.coi.2013.10.003

43. Chapuy L, Bsat M, Mehta H, Rubio M, Wakahara K, Van VQ, et al. Basophils increase in Crohn disease and ulcerative colitis and favor mesenteric lymph node memory TH17/TH1 response. J Allergy Clin Immunol. (2014) 134:978981.e1. doi: 10.1016/j.jaci.2014.05.025

44. Cassard L, Sperber K, Buivan TP, Cotillard A, Bourdet-Sicard R, Albert ML, et al. Basophils from allergic patients are neither hyperresponsive to activation signals nor hyporesponsive to inhibition signals. J Allergy Clin Immunol. (2018) 142:1548-57. doi: 10.1016/j.jaci.2017.11.053

45. Kim BS, Wang K, Siracusa MC, Saenz SA, Brestoff JR, Monticelli LA, et al. Basophils promote innate lymphoid cell responses in inflamed skin. $J$ Immunol. (2014) 193:3717-25. doi: 10.4049/jimmunol.1401307

46. Venturelli N, Lexmond WS, Ohsaki A, Nurko S, Karasuyama H, Fiebiger E, et al. Allergic skin sensitization promotes eosinophilic esophagitis through the IL-33-basophil axis in mice. J Allergy Clin Immunol. (2016) 138:1367-1380.e5. doi: $10.1016 /$ j.jaci.2016.02.034

47. Obata-Ninomiya K, Ishiwata K, Tsutsui H, Nei Y, Yoshikawa S, Kawano Y, et al. The skin is an important bulwark of acquired immunity against intestinal helminths. J Exp Med. (2013) 210:2583-95. doi: 10.1084/jem.20130761

48. Schwartz C, Eberle JU, Hoyler T, Diefenbach A, Lechmann M, Voehringer D. Opposing functions of thymic stromal lymphopoietin-responsive basophils and dendritic cells in a mouse model of atopic dermatitis. J Allergy Clin Immunol. (2016) 138:1443-1446.e8. doi: 10.1016/j.jaci.2016. 04.031

49. Noti M, Kim BS, Siracusa MC, Rak GD, Kubo M, Moghaddam AE, et al. Exposure to food allergens through inflamed skin promotes intestinal food allergy through the thymic stromal lymphopoietin-basophil axis. J Allergy Clin Immunol. (2014) 133:1390-1399.e6. doi: 10.1016/j.jaci.2014.01.021

Conflict of Interest Statement: The authors declare that the research was conducted in the absence of any commercial or financial relationships that could be construed as a potential conflict of interest.

Copyright (C) 2019 Pellefigues, Mehta, Prout, Naidoo, Yumnam, Chandler, Chappell, Filbey, Camberis and Le Gros. This is an open-access article distributed under the terms of the Creative Commons Attribution License (CC BY). The use, distribution or reproduction in other forums is permitted, provided the original author(s) and the copyright owner(s) are credited and that the original publication in this journal is cited, in accordance with accepted academic practice. No use, distribution or reproduction is permitted which does not comply with these terms. 